\title{
Actitud del personal de salud asistencial hacia las personas con VIH-SIDA, Hospital Gustavo Lanatta Luján, Bagua, Amazonas, 2017
}

\section{Attitude of health care personnel towards people with hiv / aids, Gustavo Lanatta Luján hospital, Bagua, Amazonas, 2017}

\author{
Milagritos Sánchez Chinchay ${ }^{1}$ Sonia Celedonia Huyhua Gutierrez ${ }^{2}$
}

\begin{abstract}
RESUMEN
Las personas con VIH/SIDA día a día se enfrentan al estigma y la discriminación que es parte del imaginario social incluido al personal de salud asistencial, muchas veces basados en la expresión de juicios de valor y de actitud. Cuyo objetivo fue determinar la actitud del personal de salud asistencial hacia las personas con el VIH /SIDA, Hospital Gustavo Lanatta Luján, Bagua, Amazonas, 2017. La investigación fue de enfoque cuantitativo, de nivel Descriptivo, de tipo: observacional, prospectivo, transversal, de análisis estadístico univariado. La muestra estuvo constituida por 44 profesionales; para el análisis de datos se utilizó la estadística descriptiva simple de frecuencias. Para la recolección de los datos se utilizó un instrumento denominado Escala de casos de actitud de Canales y Morales; y modificada por la autora en el 2017, obteniendo una validez: prueba binomial $=\mathrm{p}<0.05$ de 4.52 y una confiabilidad de 0.80 a través de alfa de Cronbach, además de la prueba de cálculo por Spearman Brown; los resultados fueron que la actitud del personal de salud asistencial hacia las personas con VIH/SIDA en el Hospital Gustavo Lanatta Luján en el año 2017 es que el 50\% fue positiva, 38.6 \% indiferente y el $11.4 \%$ es negativa. Conclusión la actitud del personal de salud asistencial frente a personas con VIH/SIDA es positiva.
\end{abstract}

Palabras clave: SIDA, actitud, personal de salud.

\begin{abstract}
People with HIV / AIDS every day face the stigma and discrimination that is part of the social imaginary, including health care personnel, often based on the expression of value judgments and attitude. Whose objective was to determine the attitude of health care personnel towards people with HIV / AIDS, Gustavo Lanatta Luján Hospital, Bagua, Amazonas, 2017. The research was of a quantitative, descriptive, type: observational, prospective, crosssectional approach, of univariate statistical analysis. The sample consisted of 44 professionals; For the analysis of data, simple descriptive statistics of frequencies were used. For the collection of data, an instrument called Canales de Morales attitude scale was used; and modified by the author in 2017, obtaining a validity of 4.52 and a reliability of 0.80; the results were that the attitude of the health care personnel towards people with HIV / AIDS in the Gustavo Lanatta Lujan Hospital in 2017 is that 50\% was positive, 38.6\% indifferent and 11.4\% is negative. Conclusion the attitude of the health care personnel towards people with HIV / AIDS is positive.
\end{abstract}

Keywords: AIDS, attitude, health personnel.

${ }^{1}$ Bachiller en Enfermería, egresado de la Facultad de Ciencias de la Salud de la UNTRM - A. Correo electrónico: mili10sch@hotmail.com ${ }^{2}$ Licenciada en enfermería de Escuela Profesional de Enfermería de la UNTRM - A. schg22@hotmail.com 


\section{INTRODUCCIÓN}

La Organización Mundial de la Salud mencionó que la epidemia del SIDA (Síndrome de inmunodeficiencia adquirida) continúa creciendo. Actualmente viven con el VIH alrededor de 39,5 millones de personas en el mundo. Además, alerta de un elevado crecimiento de casos en Europa del este y Asia, donde el número de infectados se ha multiplicado por 20 en los últimos 10 años (OMS, 2015, p. 34).

El VIH sigue siendo un importante problema de salud pública a nivel mundial, después de haberse cobrado más de 34 millones de vidas hasta ahora, y que la prestación insuficiente de servicios relacionados con el VIH a los grupos y poblaciones clave como: hombres que tienen relaciones sexuales con hombres, internos en instituciones penales, usuarios de drogas inyectables, trabajadores del sexo y persona transexuales pone en peligro los progresos mundiales de la respuesta al VIH (OPS, 2015, p. 47).

En el año 2016, 530 personas desarrollaron la enfermedad, mientras que el ONUSIDA señaló que en América Latina había 1,7 millones de personas afectadas por esta" (Soto, p. 24).

El Ministerio de Salud en el 2015, según la Estrategia Sanitaria Nacional de Prevención y Control de Infecciones de Trasmisión Sexual, VIH y SIDA, señale que la prevalencia del VIH en el Perú es de $0.5 \%$, es catalogada como concentrada (prevalencia en población vulnerable $>5$ por año, más de 5000 en la última década. El $80 \%$ de todos los casos son hombres y el 20\% mujeres (MINSA, 2015, p. 23).

A propósito de la relación del personal profesional de salud con los pacientes con VIH/SIDA, y que en gran parte influye en el estrés de dicho personal, es el miedo a contagiarse (Fernández, 2011, p. 54).

Para abordar esta problemática se encontraron investigaciones como Uribe, A., \& Linda, O. (2011). En su estudio encontró que la mayoría de los profesionales de la salud tienen conocimientos adecuados sobre la infección por VIH, una actitud positiva frente a las personas diagnosticadas con el virus y una mayor autoeficacia frente al VIH/SIDA.

Sin embargo Canales \& Morales, F. (2012). También demostró que en su estudio finalmente que la hipótesis de trabajo se confirma, ya que el 51.1\% del personal de salud profesional asistencial muestra una actitud desfavorable hacia las personas viviendo con el VIH/SIDA. Asimismo Altuna, H. (2011). Perú demostró que la comunicación deficiente es el principal de la interrelación del personal de salud y los pacientes viviendo con VIH/SIDA.
También ONUSIDA, menciona que las personas con VIH / SIDA se apartan por sí mismas de la asistencia que necesitan, debido al temor a reacciones negativas de los otros. El aislamiento aumenta el sentimiento de "ser el único con VIH / SIDA", y ocasiona ansiedad, depresión y suicidio debido a que su autoestima está involucrado de manera negativa (ONUSIDA, 2012, p. 34).

Amazonas ocupa el lugar 16 en el país respecto al número de casos de SIDA acumulados para el periodo 1983 - 2012. Se ha notificado al sistema de vigilancia epidemiológica hasta diciembre de 2012: 201 casos de SIDA y 167 casos de VIH. (MINSA, 2013, p. 146).

Así mismo se menciona, que las personas que padecen del VIH/SIDA no sólo tendrán que soportar un sufrimiento físico, un deterioro orgánico sino que tendrán que soportar el sufrimiento interno y el social como el miedo a la muerte, la segregación, rechazo, etc.(Colarte, 2009, p. 45).

Las Facultades de las Ciencias de la Salud hoy en día sientan sus bases en una educación holística, que integra los valores humanos al cuidado y satisface los principios bioéticos establecidos (OMS. 2015, p. 75).

Durante el tiempo del internado se ha identificado que actualmente el Hospital Gustavo Lanatta Luján tiene 298 casos o personas viviendo con VIH (+) de los cuales sólo 94 reciben tratamiento y los cuales son atendidos por el personal de salud asistencial, y toda vez que al VIH/SIDA.

Los resultados de la presente investigación servirán para sensibilizar a los profesionales de salud para que estos al estar en contacto con los usuarios infectados con el VIH-SIDA, busquen persuadirse lo que muchas de las veces sus actitudes están orientadas al aspecto más cognitivo que las emociones propias. Así mismo, complementariamente es conveniente porque desde que inició la enfermedad del VIH/SIDA poco se ha conocido sobre cual o cuales son las actitudes del personal de salud frente a esta enfermedad.

Es por ello que se decidió realizar la investigación sobre ¿Cuál es la actitud del personal de salud asistencial hacia las personas con el VIH/SIDA, Hospital Gustavo Lanatta Luján, Bagua, Amazonas, 2017.

\section{MATERIAL Y MÉTODOS}

\subsection{Tipo de investigación}

De enfoque cuantitativo porque permitió cuantificar los datos mediante el uso de la estadística. De nivel descriptivo porque describió los hechos tan igual 
como sucede en la naturaleza. Tipo de investigación: observacional porque no se manipularon las variables ya que los datos reflejaran la evolución natural de los eventos; Prospectivo porque los datos se recolectaron de fuentes primarias o sea directamente de la muestra objeto de estudio. Transversal porque las variables se midieron en una sola ocasión. De análisis univariado porque la investigación tiene una sola variable y el análisis estadístico fue el más simple de frecuencias. (Supo, 2015, p. 2).

\section{RESULTADOS}

Figura 1: Actitud del personal de salud asistencial hacia las personas con el VIH/SIDA, Hospital Gustavo Lanatta Luján, Bagua, Amazonas. 2017.

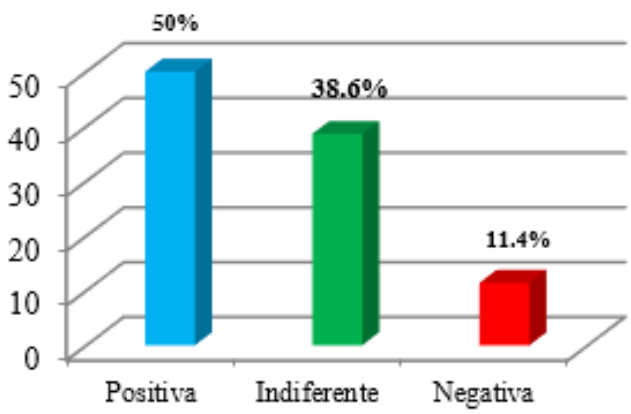

Se observa que del $100 \%$ (44) del personal de salud asistencial encuestado, el 50\% (22) presenta una actitud positiva frente al paciente con VIH/SIDA, el $38.6 \%$ (17) actitud indiferente y el $11.4 \%$ (5) actitud negativa.

Figura 2: Actitud del personal de salud asistencial hacia las personas con VIH-SIDA según dimensiones, Hospital Gustavo Lanatta Luján, Bagua, Amazonas, 2017.

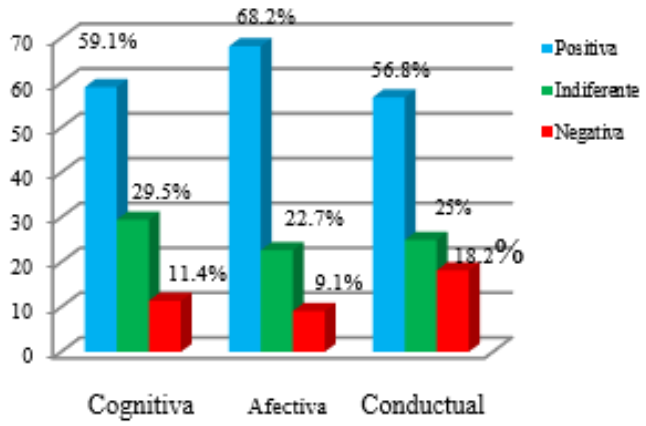

Se observa que del $100 \%$ (44) del personal de salud asistencial encuestado, respecto a la dimensión cognitiva el 59.1\% (26) presentan una actitud positiva, el 29.5\% (13) actitud indiferente y el 11.4\% (5) actitud negativa; en la dimensión afectivo el $68 \%$ (30) tienen una actitud positiva, el $22.7 \%$ (10) actitud indiferente y el $9.1 \%$ (4) actitud negativa; y en la dimensión conductual el 56.8\% (25) presentan actitud positiva, el $25 \%$ (11) actitud indiferente y el $18.2 \%(8)$ actitud negativa; respecto a las personas con VHI/SIDA.

\section{DISCUSIÓN}

En la figura 1 se observa que del 100\% (44) personal de salud asistencial en estudio el 50\% (22) presenta una actitud positiva hacia las personas con VIH/SIDA, el $38.6 \%$ (17) presenta una actitud indiferente y el $11.4 \%$ (5) presenta una actitud negativa, lo que indica que la mayoría de personal de salud asistencial presenta actitud positiva, por lo que no presentaron sentimientos negativos ni rechazo, poca confidencialidad, ni de medidas de bioseguridad exageradas.

Con respecto a la actitud según sexo, se observa que del $100 \%$ (44) del personal de salud el 27.3\% (12) son de sexo femenino y presenta una actitud positiva; el $22.7 \%$ (10) son de sexo masculino y presentan actitud positiva; el 20.5\% (9) son de sexo masculino y presenta una actitud indiferente; el 18.2\% (8) son de sexo femenino y presentan una actitud indiferente, el $6.8 \%$ (3) son de sexo masculino y presentan una actitud negativa y el $4.5 \%$ (2) son de sexo femenino y presentan una actitud negativa frente al paciente con VIH - SIDA. De estos resultados se puede deducir que la actitud no se relaciona con la edad, puesto que ambos sexos se presentó los diferentes tipos de actitud, sin mucha diferencia.

Similares resultados encontraron Uribe \& Linda (2011), en un estudio realizado en Colombia, en el cual evidencian una actitud positiva del personal de salud en su mayoría frente a las personas diagnosticadas con el virus y una mayor autoeficacia frente al VIH/SIDA.

Sin embargo, Infante et al (2006) encontró en su estudio realizado en México diferentes resultados, indicando que el $75 \%$ persiste con la discriminación o sea con una mala actitud hacia las personas con VIH/SIDA debida a clasificaciones en grupos de riesgo; la identificación de vivir con el virus, padecer el síndrome y morir; y el desconocimiento de las vías de transmisión.

Por su parte Canales \& Morales (2012) demostró que el $57.8 \%$ muestra una actitud de miedo a infectarse por la atención de la persona viviendo con el VIH/SIDA; el $48.9 \%$ rechazaría a una persona viviendo con el VIH/SIDA; el 35.6\% del personal de salud profesional asistencial le negaría la atención; el $33.3 \%$ tiene la predisposición a sentir asco hacia una persona viviendo con el VIH/SIDA; que el 51.1\% tiene la predisposición a faltar a la confidencialidad sobre la condición de una persona viviendo con el VIH/SIDA; el $48.9 \%$ tiene la predisposición de 
exagerar las medidas de bioseguridad durante la atención a las personas viviendo con el VIH/SIDA; y finalmente que la hipótesis de trabajo se confirma, ya que el $51.1 \%$ del personal de salud profesional asistencial muestra una actitud desfavorable hacia las personas viviendo con el VIH/SIDA.

Al comparar los resultados encontrados en el presente estudio y los antecedentes de estudio, algunos coinciden y otros difieren; en tal sentido las actitudes del personal de salud son variables, algunos muestran actitudes favorables o positivas y otros muestran actitudes desde indiferentes a negativas o desfavorables. En el presente estudio el 50\% presentan un actitud positiva, lo que quiere decir que de acuerdo a los contextos, según la cultura el área geográfica, creencias y sobretodo la formación profesional determina la actitud del personal de salud asistencial que atienden a personas con VIHSIDA, asimismo puede determinar el grado o nivel de cuidado que brinda a la persona con VIH- SIDA.

Según TOOD (2017) en su estudio estima que los estigmas superpuestos y estigmas relacionados con el sistema de salud deben reducirse para que las personas con VIH- SIDA puedan recibir un cuidado de calidad. Esto quiere decir que la atención de salud frente a este sistema de salud de atención debe mejorar para esto debe realizar estrategias por el bien del a población con la finalidad de brindad una atención integral y continua, a la persona familia y comunidad que viven con el virus de inmunodeficiencia adquirida.

Se puede indicar que, aun haciendo comparaciones con los antecedentes, en nuestra realidad el personal de salud asistencial tiene debilidades que mejorar respecto a la atención que se le brinda a la persona con VIH/SIDA.

En la figura 2 se observa que del 100\% (44) del personal de salud asistencial encuestado solamente presentan actitud positiva en dimensión cognitiva $(59.1 \%)$, en la dimensión afectivo (68\%), en la dimensión conductual $(56.8 \%)$ respecto a las personas con VIH/SIDA; el porcentaje restante presenta actitud indiferente y negativa, en lo que se enuncia que el personal de salud asistencial tiene una actitud más afectiva en tener menos asco y negar la atención a la persona con VIH/SIDA.

Considerando estos resultados se pude citar a Rangel (2005) en una investigación realizada en Chile, en cuyos resultados se evidencia que el trabajador de la salud que atiende personas con VIH/SIDA no siempre tiene conocimiento actualizado con respecto a la enfermedad, por lo que se forma falsas ideas las cuales repercuten en las actitudes que el trabajador tiene hacia el paciente, estas son de rechazo y discriminación, repercutiendo en la atención de calidad y calidez que requieren los portadores.

Además Rangel (2005) complementa que algunos trabajadores expresaron que evitan realizar cualquier tarea en trabajo sin usar guantes de látex por temor a infectarse de VIH/SIDA y que también evitaban tocar a usuarios, sus ropas y pertenencias. Por otro lado Arroyo (2014) evidencia en un estudio realizado en Argentina que Tanto los profesionales de la salud como los estudiantes de enfermería muestran una actitud incómoda a la hora de atender a este tipo de pacientes debido al déficit de conocimientos específicos sobre la enfermedad.

Según Carranza (2010) en un estudio ejecutado en Lima se evidencia que del 100\% (39) de profesionales de enfermería, el 77\% (30) muestran una actitud medianamente favorable, $12.8 \%$ (5) desfavorable y un $10.2 \%$ (4) favorable. De igual manera Silva et al (2001) en su estudio realizado en el Servicio de Emergencia del Hospital Guillermo Almenara Irigoyen, encontró que el 67\% tienen una aceptación favorable y el $33 \%$ tienen una actitud de rechazo, temor y se sienten mal al atender al paciente infectado con VIH.

De los resultados arribados en el presente estudio y los antecedentes revisados se puede observar que las actitudes del personal de salud y especialmente de los profesionales de enfermería son variables, en algunos contextos se encontró actitudes más favorables que en otros, por tanto sería importante profundizar los estudios para determinar las actitudes predominantes del personal de salud con la finalidad de proponer alternativas de solución y mejorar la atención del paciente con VIH/ SIDA considerando que la prevalencia de esta enfermedad va en aumento y más aún en la población de la región Amazonas.

En tal sentido es necesario recordar que según Antela (2004) el Síndrome de inmunodeficiencia adquirida (SIDA), es definido como el conjunto de manifestaciones clínicas que aparecen como consecuencia de la depresión del sistema inmunológico debido a la infección por el virus de la inmunodeficiencia humana (VIH). Una persona infectada con el VIH va perdiendo, de forma progresiva, la función de ciertas células del sistema inmune llamadas linfocitos T CD4, lo que la hace susceptible a desarrollar cierto tipo de tumores y a padecer infecciones oportunistas.

Se observa que le personal de salud teme al atender al paciente con VIH/SIDA ya que tiene miedo al contagio de esta letal enfermedad, asimismo la actitud de acuerdo a las dimensiones no es la misma o 
la adecuada, los resultados con esta investigación difieren ya que en estas investigaciones los autores demuestran un alto índice de rechazo frente a la atención debido al temor, la discriminación de alejarlo de la comunidad.

Se encuentra en discrepancia debido a que sabemos que una persona con VIH/SIDA es una persona susceptible es por ello que debemos cuidarle y no exponerles a ciertos factores de riesgo, pero también se trata de brindar un cuidado holístico, de manera físico y psicológica; que al aislarlo podemos dañarlo cognitiva afectiva y conductualmente, ahora la actitud no es del personal de salud si no del paciente que se va sentir rechazado.

Según Altuna, H. (2011). En el Perú existen problemas en la relación establecida con el personal de salud, la concepción existente del derecho a la atención en salud, y la calidez en el servicio; siendo esta última la que posee mayores problemas, lo que limita la adherencia de las personas viviendo con VIH al tratamiento, y así, pone en riesgo el objetivo perseguido por la Estrategia. En cuanto a la actitud del personal de salud, el 75\% tienen una actitud negativa de parte del sexo masculino de indiferencia hacia las personas viviendo con VIH/SIDA, el 25\% tiene una actitud positiva de parte de las mujeres.

Todo ello se basa en reflexionar y mejorar las actitudes respecto a las dimensiones o comportamientos, porque el ser humano su actuar está constituida por tres factores, el pensamiento, las emociones y sensaciones, ya que cada movimiento responde a nuestra percepción de los estímulos externos para poder enfrentar las situaciones de salud que se presenten.

Lo ideal que se debe lograr es que todo el personal de salud asistencial en su $100 \%$ debería tener una actitud favorable en la atención hacia la persona con $\mathrm{VIH} / \mathrm{SIDA}$, por ende es necesario que se elaboren estrategias y talleres al personal, con el fin de mejorar los servicios de salud, y que haya una continua supervisión al personal del salud en la atención del paciente con VIH/SIDA; para así en un futuro lograr brindar un servicio sin discriminación.

\section{CONCLUSIÓN}

La mayoría del personal de salud asistencial del Hospital Gustavo Lanatta Lujan, muestran una actitud positiva frente al paciente con VIH/SIDA en cuanto se refiere a su atención; sin embargo existe un porcentaje considerable que se tiene una actitud indiferente y negativa.

\section{REFERENCIAS BIBLIOGRÁFICAS}

Altuna, H. (2011). Problemas y actitudes de las personas viviendo con VIH frente a la atención en TARGA: Derechos, ciudadanía y comunicación. Tesis para optar el título de Licenciada en Ciencias y Artes de la comunicación. Universidad Pontificia la Católica, Perú.

Antela, A. (2004). Manual de capacitación en el manejo integral de personas adultas que viven con el VIH/SIDA para equipos de atención primaria y comunitaria en Latinoamérica y el Caribe. Washington, D.C.: Organización Panamericana de la Salud.

Canales, Y., \& Morales, F. (2012). Perú. Actitud del personal de Salud profesional asistencial hacia las personas viviendo con el VIH/SIDA del hospital II -2

Carranza, R. (2010). Actitudes de las enfermeras hacia la atención del paciente VIH/SIDA en el servicio de emergencia Hospital Nacional Edgardo Rebagliati Martins. Lima: accesado el 25 de enero 2018 . http://ateneo.unmsm.edu.pe/ateneo/bitstrea m/123456789/3458/1/Carranza_Bullon_R ocio_del_Pilar_2010.pdf

Colarte, A. (2009). La enfermedad del siglo?. El SIDA y formas de prevenirlo".3era edición, Amorrortu. Barcelona. España.

Fernández, E. (2011). “Guía de prevención de la transmisión del VIH para mediadores y mediadoras".1era edición, Eudema. Madrid. España.

Infante, C., et al. (2006). El estigma asociado al VIH/SIDA: el caso de los prestadores de servicios de salud en México. Recuperado e $1 \quad 15$ d e julio d e 2015 d e http://www.unaids.org/es/PolicyAndPracti ce/HumanRights/default.asp

MINSA. (2013). Análisis de la situación epidemiológica del VIH/SIDA en el Perú, pg. 146. Recuperado el 14 julio del 2016 de http://bvs.minsa.gob.pe:81/local/2740.pdf.

OMS. (2015). VIH/SIDA, recuperado el 14 de julio $\mathrm{d} \mathrm{e} \quad 2016, \quad \mathrm{de}$ http://www.who.int/mediacentre/factsheets /fs360/es/

ONUSIDA. (2012). "Violaciones de los derechos humanos, estigma y discriminación" recuperado el 15 de Julio del 2016. 
http://whqlibdoc.who.int/hq/2004/OMS HTM_TUB_2004.339_spa.pdf>.

Rangel, L. (2005). "El personal de salud ante la atención de los pacientes con VIH/SIDA. El caso del Hospital Integral de Jalca Hidalgo". Chile: accesado el 25 de enero d e $1 \quad 20018$. https://repository.uaeh.edu.mx/bitstream/b itstream/handle/123456789/1 1229/E\%20p ersonal $\% 20 \mathrm{de} \% 20$ salud $\% 20$ ante $\% 201 \mathrm{a} \% 2$ 0atenci\%C3\%B3n\%20de $\% 201$ los\%20paci entes $\% 20$ con $\% 20$ VIHSida. $\% 20$ Caso $\% 20$ del $\% 20$ Hospital\%20Integral $\% 20 \mathrm{de} \% 20 \mathrm{Ja}$ cala $\% 20$ Hidalgo..pdf? sequence $=1$

Silva, M., et al. (2001). Actitudes del equipo de salud hacia la atención del paciente con VIH/SIDA en el servicio de emergencia del Hospital Guillermo Almenara Irigoyen. Accesado el 25 de enero 2018 . file://D:/bibliografia\%20adicional/Actitud es $\% 20 \mathrm{del} \% 20$ equipo $\% 20 \mathrm{de} \% 20$ Salud $\% 2$ 0hacia $\% 201 \mathrm{a} \% 20$ atenci $\% 3 \%$ B3n $\% 20 \mathrm{del}$ $\% 20$ paciente $\% 20$ con\%20HIV.html

Supo, J. (2015). "Metodología de la investigación científica para las ciencias de la salud". 2da Edic. Bioestadística. Arequipa.

Soto, L. (2008). Manual del usuario del equipo multidisciplinario que brinda TARGA. 2008. Lima: Instituto de medicina tropical "Alexander Von Humboldt" - Universidad Peruana Cayetano Heredia. Consulta: 9 de septiembre de 2009.

Tood, R. (2017). "Estigmas superpuestos y estigmas relacionados con el sistema de salud debe reducirse para que las personas con VIHSIDA puedan recibir un cuidado de calidad". Accesado el 18 de noviembre del 20017 d e h t t p : / / w w w . Estigmas_superpuestos_y_estigama.com. pe.

Uribe, A. \& Linda, O. (2011). Evaluación de conocimientos, actitudes, susceptibilidad y autoeficacia frente al VIH/sida en profesionales de la salud. Recuperado el 15 d e ju 1 i o d e 2016 d e http://www.scielo.org.co/pdf/aven/v29n2/ v29n2a07 